\title{
Assessment of motion of colonic contents in the human colon using MRI tagging.
}

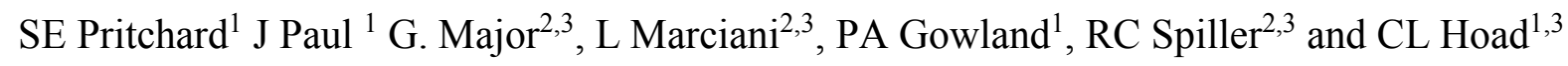

${ }^{1}$ Sir Peter Mansfield Imaging Centre, School of Physics and Astronomy, University of Nottingham, Nottingham, UK

${ }^{2}$ Nottingham Digestive Diseases Centre, School of Medicine, University of Nottingham, Nottingham, UK

${ }^{3}$ NIHR Biomedical Research Unit in Gastrointestinal and Liver Diseases at Nottingham University Hospitals NHS Trust and The University of Nottingham, Nottingham, UK

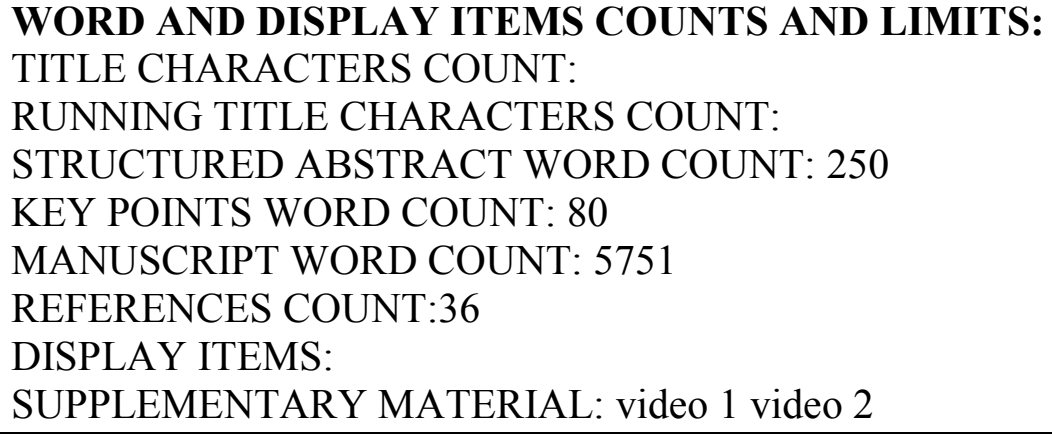

Running title: MRI assessment of colonic content flow.

\section{Correspondence:}

Dr Caroline Hoad

Sir Peter Mansfield Imaging Centre

School of Physics and Astronomy

University Park

University of Nottingham

Nottingham NG7 2RD, U.K.

Email: Caroline.L.Hoad@nottingham.ac.uk 
2 ABSTRACT

3 Background We have previously reported a non-invasive, semi-automated technique to 4 assess motility of the wall of the ascending colon (AC) using Magnetic Resonance Imaging.

5 This study investigated the feasibility of using a tagged MRI technique to visualise and assess

6 the degree of flow within the human ascending colon in healthy subjects and those suffering

7 from constipation.

8 Methods An open-labelled study of 11 subjects with constipation and 11 subjects without 9 bowel disorders was performed. MRI scans were acquired fasted, then 60 and 120 mins after 10 ingestion of a 500ml macrogol preparation. The amount of free fluid in the small and large 11 bowel was assessed using a heavily T2-weighted MRI sequence. The internal movement of the contents of the AC were visualised using a cine tagged MRI sequence and assessed by a novel analysis technique. Comparisons were made between fasting and postprandial scans within individuals, and between the constipation and control groups.

Key results. Macrogol significantly increased the mobile, MR visible water content of the ascending colon at 60 mins post ingestion compared to fasted data (controls $p=0.001$, constipated group $\mathrm{p}=0.0039$ ). The contents of the $\mathrm{AC}$ showed increased motion in healthy subjects but not in the constipated group with significant differences between groups at 60 minutes $(\mathrm{p}<0.002)$ and 120 minutes $(\mathrm{p}<0.003)$.

Conclusions and inferences. This study successfully demonstrated the use of a novel MRI tagging technique to visualise and assess the motion of ascending colon contents following a $500 \mathrm{ml}$ macrogol challenge. Significant differences were demonstrated between healthy and constipated subjects. 
Pritchard 3

28 KEY POINTS:

29 Manometry techniques provide information about pressure changes that occur when the colon

30 wall contracts. There is little knowledge about how the contents move.

31

32 Using an MRI tagging technique we showed differences in the movement of colonic chyme

33 (following a macrogol stimulus) between subjects with constipation and healthy controls.

35 This non-invasive MRI technique has wide application as a tool to investigate the movement

36 of colonic contents in constipation and diarrhoea to help further our understanding of the 37 physiology of the colon. 
INTRODUCTION

40 Constipation is a common problem worldwide, with estimates of prevalence ranging from $14 \%$ to $28 \%$ in the USA [1] and associated annual health care costs recently estimated to exceed \$230 million [2]. Generally constipation disorders have been categorised in three ways [3]: firstly, constipation arising from disordered or obstructed defecation (OC) (where the primary cause is impaired rectal evacuation) [4], secondly irritable bowel syndrome with constipation (IBS-C) accompanied by pain and bloating, often with transit times within the wide range of normality, and thirdly, functional slow transit constipation (FC). As we have recently shown, although the symptoms overlap substantially, these conditions have different mechanisms of disease [5] and may require different treatments. Distinguishing these conditions based mostly on patient perception leads to trial and error treatments which may explain why $50 \%$ of patients are dissatisfied with their treatment [6].

We showed previously that, while baseline measurements do differ, the contrast between patient groups can be enhanced by stressing the colon using an osmotic laxative to distend the ascending colon, so that 1 hour after ingestion of 1 litre of a macrogol drink, colonic volumes become abnormal in 19/20 FC patients [7].

In our previous work, using cine MRI to determine colonic wall motility, it was clear that there was also motion occurring within the colonic contents, probably related to the gross movement and mixing of these contents. MRI tagging is commonly used for the assessment of cardiac function and has been applied previously to monitor small bowel motility $[8,9]$ and to study movement of the stomach contents following a porridge meal [10].

In this study, we aimed to investigate whether an MRI tagging technique could be used to assess movement within in the human ascending colon and to differentiate between the colonic response to a $500 \mathrm{ml}$ macrogol oral stimulus in healthy and constipated subjects. 
METHODS

66

67

68

69

\section{Subjects and study design}

The study protocol was approved by the Local Research Ethics Committee (control group: G08052014 SoM NDDC and constipated group: J14082014 SoM NDDC). Twenty two subjects were recruited by advertisement and from a database of subjects who had taken part in previous studies and had agreed to be contacted again. All subjects gave written informed consent and had no contraindications to MRI. There were no adverse events during the studies.

The 11 healthy participants had no history of gastrointestinal disease. There were 4 males and 7 females, aged $28 \pm 10$ years (mean \pm standard deviation) with mean Body Mass Index (BMI) $25 \pm 5 \mathrm{~kg} \mathrm{~m}^{-2}$. The 11 constipated participants (as defined by Rome III criteria) were 2 males and 9 females aged $36 \pm 13$ years, with BMI $25 \pm 5 \mathrm{~kg} \mathrm{~m}^{-2}$. Constipated subjects were asked to refrain from taking their usual laxatives for two days before the MRI study day. All subjects ingested 5 MRI transit marker capsules as described previously [11, 12] 24 hours prior to MRI. Subjects were asked to avoid strenuous exercise and ingesting alcohol and caffeine the day before their attendance for MRI, and to fast from 22:00 hours the previous evening. They were only allowed to consume a small glass of water on waking on the day of the experiment. Participants completed a questionnaire to investigate adherence to the study restrictions before acquiring the baseline, fasted MRI scan. They were then asked to drink a $500 \mathrm{ml}$ dose of MOVIPREP ${ }^{\circledR}$ (Norgine Pharmaceuticals Ltd, Harefield, UK) polyethylene glycol (Macrogol 3550) electrolyte solution within 30 minutes. The time the subjects started consuming the test drink was defined as $\mathrm{t}=0$. This was followed by a scan at $\mathrm{t}=60 \mathrm{mins}$ and $\mathrm{t}=120$ mins. 
90 All MRI scans were carried out using a 3T Philips Achieva scanner (Philips, Best, The

91 Netherlands). The subjects were positioned supine with a 16-channel XL-torso receiver coil wrapped around their abdomen. After the initial set-up scans, the following scans were acquired across the abdomen:-

(1) A multi-echo mDIXON scan [13] was used to determine the location of the transit pills and calculate a weighted average position score (WAPS) for each subject (a validated measure of whole gut transit time) $[11,12]$.

(2) A T2-weighted single shot (RARE) sequence was used to determine the mobile, MR visible water content of both the small bowel (Small Bowel Water Content, SBWC) and colon [14-17]. This sequence was included to enable monitoring of the progress of the

100 laxative drink through the GI tract.

101 (3) To aid in the positioning of the tagged slice through the AC a high resolution multi-slice bTFE scan was acquired, placed sagitally oblique through the AC [16].

(4) Motion of the contents of the AC were visualized using a tagged bTFE sequence [9]

104 centered within the ascending colon (primarily sagittal). This sequence superimposed dark 105 horizontal stripes (tags), $12 \mathrm{~mm}$ apart, onto the images. The delay of $250 \mathrm{~ms}$ between application of the tag lines and acquisition of the image allowed movement within the colon

107 to be detected (see Fig. 1). This sequence had TR/TE 2.3/1.15 ms, FA $45^{\circ}$, with a single 108 sagittal slice, thickness $15 \mathrm{~mm}$, FOV 222-264 mm (AP), $330 \mathrm{~mm}$ (HF) with acquired 109 resolution of $1.5 \times 1.5 \mathrm{~mm}^{2}$ and reconstructed to $0.98 \times 0.98 \mathrm{~mm}^{2}$, SENSE factor 1.5 , and half110 scan factor 0.7. In total, 33 dynamic scans were acquired at $600 \mathrm{~ms}$ intervals within a single 11120 s breath-hold.

112 All other imaging parameters associated with the different sequences are summarized in 113 Table (1). 
114 The subjects spent 30 minutes per time point inside the scanner and they were asked to spend

115 the rest of the time sitting upright in an adjacent room.

\section{Data analysis}

118 An overall assessment of gut transit time (WAPS) was obtained by noting the position of 5

119 marker capsules ingested $24 \mathrm{hrs}$ prior to scanning as previously reported $[11,12]$. The free 120 water in the small bowel (SBWC) and colon were measured using the RARE sequence as 121 previously described $[14,18]$.

123 The series of tagged images of the ascending colon provided a method to visualize motion 124 within the colon and were also used to assess the movement of the colon contents. If no movement of colonic contents, or adjacent viscera, occurred during the breath hold (eg due to small respiratory movements or aortic pulsation) then all 33 sequential tagged images would

127 be identical, with all tag lines remaining straight (as seen in Fig 1a). However any movement

128 of the colonic chyme in the delay between the application of the tag and acquisition of the 129 image would change the position of the tag lines on the image. For instance, if laminar flow was present then each frame would show uniform displacement of each tag line within the colon, with the displacement proportional to the flow velocity, $12 \mathrm{~mm}$ corresponding to a

132 flow velocity of $4.8 \mathrm{~cm} / \mathrm{sec}$ in this case. Laminar flow velocities greater than this value 133 would not be detected due to the periodicity of the tag lines. The predominant direction of

134 flow (antegrade or retrograde) would also be revealed from the antegrade (arrowed) or retrograde direction of displacement of the tagged lines (as can be seen in Fig 1b ) Therefore

136 the movement of the tags could, in principle, be used to measure laminar flow velocity.

137 However, as can be seen in Fig. 2, non-laminar flow leads to local variations in the 3D 138 velocity field which can smear the tag and prevent absolute velocity measurement. This 
139 provides a simple method to assess movement of the colonic content, since motion, in any

140 direction, will lead to changes in signal intensity in the tagged colonic contents from frame to

141 frame. It is this variation in intensity which is the basis of the proposed method of analysing

142 the data to assess motion within the colonic contents.

144 The first 4 frames of each sequence were discarded due to intensity changes occurring as the 145 MRI signal reached steady state. The mean signal intensity $(\mathrm{MI}(\mathrm{x}, \mathrm{y}))$ and standard deviation $146(\operatorname{STDEV}(\mathrm{x}, \mathrm{y}))$ of each pixel through the remaining 29 sequential cine frames were calculated 147 using IDL $^{\circledR}$ (Research Systems Inc, Boulder, CO) resulting in maps of both mean intensity 148 and standard deviation (Figure $2 \mathrm{a}$ and $\mathrm{b}$ ).

150 In the standard deviation maps those voxels whose intensity is changed during the 20 seconds

151 of data acquisition (either by the movement of structures such as the colon wall, or by the 152 movement or smearing of the null tagged lines) have a larger standard deviation (Fig. 2b) 153 whereas static structures (liver, muscle and fat) or motionless colonic contents have a value 154 close to zero (Fig. 2a). The resulting map thus highlights any motion of the colonic contents, even where the net displacement over 20 seconds could be zero. This standard deviation map also reveals where the motion is concentrated eg if it is the whole colon that has moved then

157 all the contents will show a high standard deviation whereas movement in a narrow 'jet' will 158 lead to a smaller region of high intensity within the colon. In order to derive a single 159 parameter that easily summarises this motion of the colonic contents a region encompassing 160 the $\mathrm{AC}$ was drawn on the mean intensity map, as shown in figure 2, and the average mean 161 intensity $\left(\mathrm{MI}_{\mathrm{R}}\right)$ and average $\operatorname{STDEV}\left(\mathrm{STDEV}_{\mathrm{R}}\right)$ within that region was calculated (using 162 Analyze9 ${ }^{\mathrm{TM}}$, Mayo Foundation, Rochester, MN,USA). The average coefficient of variation $163(\% \mathrm{COV})$ for the tagged scan is then estimated from 
Statistics

167 Normality of the data was tested using D'Agostino Pearson's normality test and the data are 168 expressed as mean, (standard deviation) for normally distributed data and median values 169 (with the IQR indicated in brackets), for non-normally distributed data. Statistical analysis was carried out using Prism 6 (GraphPad Software Inc.). Comparisons within group were performed using a paired t-test (for normally distributed data) or Wilcoxon's matched-pairs signed rank test, for data non-normally distributed. Comparisons between groups were

173 performed using an unpaired t-test (with Welches correction for normally distributed data) or

174 Mann-Whitney rank sum test for non-normally distributed data. Multiple comparisons of data were Bonferroni corrected, with comparison between baseline and 60, baseline and 120, for both cohorts and at all times between groups resulting in a corrected significant $p$-value of $<$

$177 \quad 0.0071$

\section{RESULTS}

180 All subjects completed the study day. MRI scans were not available for two constipated 181 subjects at $\mathrm{t}=60$ mins, one healthy volunteer at $\mathrm{t}=60$ mins and one healthy volunteer at $\mathrm{t}=120$

182 mins due to equipment problems.

\section{Whole Gut Transit}

185 At baseline the WAPS measured using the MR markers were significantly different between

186 groups: controls $0.6(0-1)$ and constipated group 2.6 (1.4-3.6), $\mathrm{p}=0.0011$ Mann-Whitney rank 187 sum test. 
189 The amount of mobile, free water in the small bowel (SBWC) is shown in Fig. 3a. There was

190 no significant difference in the fasted baseline values of these groups (controls $33(22-89) \mathrm{ml}$,

191 constipated group $66(42-124) \mathrm{ml} \mathrm{p}=0.11)$. Sixty minutes after macrogol ingestion, the

192 SBWC was significantly increased in both groups, (controls 372 (220-566) ml p=0.001,

193 constipated group 472 (337-708) $\mathrm{ml} \mathrm{p}=0.0039$; Wilcoxon ranked pairs test). By $\mathrm{t}=120$

194 minutes SBWC had dropped in both groups (controls 231 (157-322) ml, constipated group

$195406(215-517) \mathrm{ml})$, although the change from baseline remained significant for both groups

196 (controls $\mathrm{p}=0.0068$, constipated group $\mathrm{p}=0.001$ Wilcoxon ranked pairs test), when multiple

197 comparisons were taken into account. However there were no significant differences in

198 SBWC between groups at $60(\mathrm{p}=0.41)$ and 120 mins $(\mathrm{p}=0.09)$ (Mann-Whitney rank sum test).

200 The variation in free water content in the ascending colon is shown in Fig. 3b. There was no significant difference in the very low baseline values between groups (controls $2(0-7) \mathrm{ml}$, constipated group $11(1-29) \mathrm{ml} \mathrm{p}=0.16)$. By 60 mins post ingestion the amount of mobile,

203 free water was significantly increased in the healthy control groups, (140 (104-347) ml,

$204 \mathrm{p}=0.001$ ), and in the constipated group 228 (91-259) $\mathrm{ml}, \mathrm{p}=0.0039$ (Wilcoxon ranked pairs test) showing that the macrogol solution had entered the ascending colon in both groups. By 120 minutes post ingestion the amount of mobile, free water in the ascending colon had only dropped in the constipated group (controls 146 (32-227) ml, constipated group 84 (3-195) ml) although the change from baseline was still significant in both groups, (controls $p=0.002$, constipated group $\mathrm{p}=0.0039)$ when corrected for multiple comparisons. Again there were no significant differences in the free water in the ascending colon between groups at $60(p=0.66)$ or at 120 mins $(\mathrm{p}=0.24)$ (Mann-Whitney rank sum test). 
214 Baseline sequences showed intact tags (as shown in Figs. 1a, 2a and supplementary

215 information video 1) indicating very little motion within the colon in both groups. After 60

216 mins displacement and smearing of the tags (as shown in Fig. 1b) was observed in some

217 subjects (mainly healthy volunteers). Fig. 4a shows the presence of forward and backward tag

218 displacement occurring simultaneously in the central regions of the ascending colon. Fig. 4b

219 demonstrates the presence of a fast $(>4.8 \mathrm{~cm} / \mathrm{sec})$ retrograde central 'jet', which was also observed (supplementary information video 2). Tag smearing resulting from non-1D laminar flow can be seen as a reduction of tag intensity in Fig. 4c. Increased movement in the central regions of the ascending colon, compared to that adjacent to the colon walls, (seen in all parts of Fig. 4) was observed throughout the data sets. Such motion was still visible at $t=120$ minutes, although predominantly in the healthy subjects rather than the constipated group.

The calculated average coefficient of variation $(\% \mathrm{COV})$ for the all the completed tagged scans of the ascending colon is shown in Fig. 5. As expected there is no significant difference in the low baseline values between groups (controls 20\% (14-23), constipated group 12\% $(11-20) ; \mathrm{p}=0.1)$. By 60 mins post ingestion the $\% \mathrm{COV}$ was significantly increased in the control group only $(30 \%(26-35), \mathrm{p}=0.002$; constipated group 17\% (13-23), $\mathrm{p}=0.57$; Wilcoxon ranked pairs test). By 120 minutes the \%COV had dropped in both groups but the change from baseline remained significant only for controls (controls $25 \%(18-36), p=0.002$; constipated group $13 . \%(12-18), \mathrm{p}=0.76)$. This led to a significant difference in the $\% \mathrm{COV}$ between groups at 60 mins ( $\mathrm{p}=0.002)$ and $120 \mathrm{mins}(\mathrm{p}=0.003)$ (Mann-Whitney rank sum test).

\section{DISCUSSION}

237 MRI tagging and our proposed analysis technique provide a new method to visualize 
239 been used to demonstrate differences between healthy and constipated subjects in response to

240 a 500ml macrogol challenge.

241 MRI is an ideal tool to study the physiology of the bowel, allowing assessment of changes in

242 whole gut transit, contractile activity, and fluid distribution both in the small bowel $[9,14,15$,

243 19-30] and the colon [11, 12, 21, 22, 31-33] during interventional studies. The whole bowel

244 (small and large) can be assessed in a single scanning session and, since MRI is non-invasive, repeated studies are possible. The additional information gained from the tagging sequence provides further insights into how the chyme moves within the large bowel. Specifically the proposed $\% \mathrm{COV}$ measure relates to the movement and blurring of the tags and therefore mixing related motion. More sophisticated analysis of this tagged data might allow more detailed assessment of the velocity fields within the colon. Additional blurring of the tag lines could arise from motion occurring during the readout phase of the acquisition and will have contributed to the variance measured here. In future alternative methods of analysis providing more quantitative measures will be investigated. Phase contrast MRI (PC-MRI) is the conventional method of measuring flow but is less suited to measuring flow in the colon for two reasons. Firstly, tagging is less sensitive to overall subject motion than PC-MRI since the tag lines are inherently sensitive to local motion, not bulk motion. Secondly, tagging will lose sensitivity if the $T_{1}$ of the material being scanned is too short as the lines will recover

257 (and hence disappear) in the delay period. PC-MRI will lose sensitivity if the $\mathrm{T}_{2}$ of the sample is short as the signal will decay during the phase encoding period. In practice the MRI properties of normal colonic contents are likely to favour the use of tagging over PC-MRI.

260 Finally, tagging provides an immediate assessment of the motion which would be useful for non-expert sites.

262 A central channel of increased displacement was seen on the majority of healthy volunteers' 263 data, with the haustra probably impeding flow near the walls of the colon. This may be a 
264 biological mechanism to reduce the impact of the axial fluid flow on the anaerobicity of the more static chyme adjacent to the colon walls (where anaerobic microbiota may escape poisoning by oxygenated ileal contents entering the colon), thus preserving the mixture of aerobic and anaerobic microbiota essential for health.

268 This study demonstrated clear changes in SBWC, ascending colon water and motion of the 269 chyme in the ascending colon of 11 healthy volunteers at 60 mins and 120 mins following the $270500 \mathrm{ml}$ macrogol challenge drink, a smaller stimulus than has previously been used [34]. This

271 indicates that relatively moderate quantities of fluid passing through the small bowel and 272 arriving in the ascending colon can trigger wall contractions and movement of the colon 273 contents in health. However, although the SBWC and ascending colon water was also 274 increased in the constipated group at $60 \mathrm{mins}$ and $120 \mathrm{mins}$ following the challenge, the motion of the contents in the ascending colon (as assessed by \%COV) was not increased in this group post-ingestion. This lack of motion led to a significant difference in $\%$ COV being observed between groups at both 60 and 120 mins post-ingestion. This suggests that this parameter may successfully discriminate between healthy and constipated subjects when the ascending colon is challenged appropriately.

281 The motility of the descending and sigmoid colon has been previously studied using manometric techniques and these have demonstrated differences in contractile activity 283 present in IBS-C and in chronic slow transit constipation [33, 35]. IBS-C patients have 284 normal, or even excess, antegrade and retrograde contractile activity (but this activity fails to move the contents through the colon) whereas FC patients frequently have much lower levels of contractions. However, manometry is invasive and often does not monitor the entire colonic region (particularly the proximal ascending colon where much of the mixing processes occur), and as a result is limited clinically to extreme cases, particularly for 
paediatric populations. Manometry cannot be used to study movement of the contents of a

290 fluid filled colon as the pressure measurements become less accurate when the colonic

291 contents become less viscous [36] and tracking of contents is not possible so that manometry

292 cannot directly detect mixing.

294 The main limitations of this study were the small numbers in each group. In addition, the

295 smaller macrogol challenge drink may not have stimulated the same type of motion seen 296 previously with a larger challenge drink. This smaller fluid stimulus was also less likely to

297 reach the more distal parts of the colon (descending and sigmoid) and hence may be less

298 suitable to differentiate between subgroups of constipation patients.

300 As the motion and mixing observed in this study was due to the stimulus of the laxative drink,

301 data on the reproducibility of the measurement would be extremely useful and work is underway to obtain this data. Further work is required to fully explore the potential of MRI tagging for assessment of mixing and transportation of colonic chyme including other

304 possible physiological challenges such as a high fat meal. This potential ability to monitor 305 non-invasively both antegrade and retrograde flow patterns could be used to assess the 306 efficiency with which the ascending colon mixes and transports the contents and has 307 applications in functional disorders of both constipation and diarrhoea. Allowing for data acquisition during free breathing, and using image registration techniques to remove the

309 effects of respiration, could extend the application of this technique and allow for more

310 sporadic motion to be investigated. Combining this technique with existing motility and

311 volume measurements could provide increased discrimination between healthy subjects, 312 subjects whose contractions are ineffectual at mixing and propelling the colonic contents, and 
313 subjects in whom colonic contractions are absent, potentially allowing stratification of IBS

314 type disorders from FC.

315 In conclusion this study has demonstrated the use of MR tagging and a novel analysis method

316 to study movement of the colonic content and has used this to demonstrate significant

317 differences in the transport and mixing of colonic chyme between healthy volunteers and

318 constipation subjects following a macrogol challenge drink.

319

320 ACKNOWLEDGEMENTS

321 We are grateful for support from the Nottingham Digestive Diseases Biomedical Research

322 Unit. The views expressed are those of the authors and not necessarily those of the NHS, the

323 NIHR or the Department of Health.

326 FUNDING

327 This study was funded by an MRC Confidence in Concept Grant no. MC_PC_13072.

328

329 COMPETING INTERESTS

330 RCS has received research funding from Lesaffre and Ironwood and free drugs for clinical

331 trial from Norgine. He has also acted on Advisory Boards for Almirall, Astellas, Ibsen and

332 Danone. All other authors have no competing interests. 
335 Research was designed by CLH, LM, GM, PAG, and RCS and performed by JP, GM, and 336 CLH. It was analyzed by SEP, JP, CLH and the paper was written by SEP, CLH, GM, LM, 337 PAG and RCS. All authors read and approved the final manuscript.

338

339 
341 1. Suares NC, Ford AC. Prevalence of, and risk factors for, chronic idiopathic

342 constipation in the community: systematic review and meta-analysis. The American journal 343 of gastroenterology 2011; 106: 1582-1591.

344 2. Bharucha AE, Pemberton JH, Locke GR, 3rd. American Gastroenterological Association technical review on constipation. Gastroenterology 2013; 144: 218-238.

3. Longstreth GF, Thompson WG, Chey WD, Houghton LA, Mearin F, Spiller RC. Functional bowel disorders. Gastroenterology 2006; 130: 1480-1491.

4. Bharucha AE, Wald A, Enck P, Rao S. Functional anorectal disorders. Gastroenterology 2006; 130: 1510-1518.

350 5. Lam C, Chaddock G, Marciani L, Costigan C, Paul J, Cox E, Hoad C, Menys A, Pritchard S, Garsed K, Taylor S, Atkinson D, Gowland P, Spiller R. Colonic response to laxative ingestion as assessed by MRI differs in constipated irritable bowel syndrome compared to functional constipation. Neurogastroenterology \& Motility 2016; 28: 861-870. 6. Johanson JF, Kralstein J. Chronic constipation: a survey of the patient perspective. Alimentary Pharmacology and Therapeutics 2007; 25: 599-608.

356 7. Lam C, Chaddock G, Hoad C, Costigan C, Cox E, Pritchard S, Garsed K, Marciani L,

357 Gowland P, Spiller R. The Macrogol Drink Test to Distinguish Functional Constipation (Fc) and Constipation Predominant Irritable Bowel Syndrome (Ibs-C): Underlying Mechanisms Demonstrated Using Mri. Gut 2014; 63: A195-A195.

360 8. Sprengers AMJ, van der Paardt MP, Zijta FM, Caan MWA, Lamerichs RM,

361 Nederveen AJ, Stoker J. Use of continuously MR tagged imaging for automated motion

362 assessment in the abdomen: A feasibility study. Journal of Magnetic Resonance Imaging 363 2012; 36: 492-497.

364 9. van der Paardt MP, Sprengers AMJ, Zijta FM, Lamerichs R, Nederveen AJ, Stoker J. Noninvasive Automated Motion Assessment of Intestinal Motility by Continuously Tagged MR Imaging. Journal of Magnetic Resonance Imaging 2014; 39: 9-16.

10. Issa B, Freeman A, Boulby P, Wright J, Gowland P, Bowtell R, Spiller R, Mansfield P. Gastric motility by tagged EPI. Magnetic Resonance Materials in Physics Biology and Medicine 1994; 2: 295-298.

11. Lam C, Chaddock G, Hoad CL, Costigan C, Cox E, Marciani L, Gowland PA, Spiller RC. A new validated MRI method for measuring whole gut transit time. Gastroentrology 2013; 144: S-920.

373 12. Chaddock G, Lam C, Hoad CL, Costigan C, Cox EF, Placidi E, Thexton I, Wright J, 374 Blackshaw PE, Perkins AC, Marciani L, Gowland PA, Spiller RC. Novel MRI tests of orocecal transit time and whole gut transit time: studies in normal subjects. Neurogastroenterology and Motility 2014; 26: 205-214.

13. Eggers H, Brendel B, Duijndam A, Herigault G. Dual-echo Dixon imaging with flexible choice of echo times. Magnetic Resonance in Medicine 2011; 65: 96-107.

14. Hoad CL, Marciani L, Foley S, Totman JJ, Wright J, Bush D, Cox EF, Campbell E, Spiller RC, Gowland PA. Non-invasive quantification of small bowel water content by MRI: a validation study. Phys Med Biol 2007; 52: 6909-6922.

15. Marciani L, Cox EF, Hoad CL, Pritchard S, Totman JJ, Foley S, Mistry A, Evans S, Gowland PA, Spiller RC. Postprandial changes in small bowel water content in healthy subjects and patients with irritable bowel syndrome. Gastroenterology 2010; 138: 469-477.

16. Placidi E, Marciani L, Hoad CL, Napolitano A, Garsed KC, Pritchard SE, Cox EF, Costigan C, Spiller RC, Gowland PA. The effects of loperamide, or loperamide plus simethicone, on the distribution of gut water as assessed by MRI in a mannitol model of 
17. Mudie DM, Murray K, Hoad CL, Pritchard SE, Garnett MC, Amidon GL, Gowland

390 PA, Spiller RC, Amidon GE, Marciani L. Quantification of Gastrointestinal Liquid Volumes and Distribution Following a $240 \mathrm{~mL}$ Dose of Water in the Fasted State. Mol Pharmaceut 2014; 11: 3039-3047.

18. Murray K, Wilkinson-Smith V, Hoad C, Costigan C, Cox E, Lam C, Marciani L, Gowland P, Spiller RC. Differential Effects of FODMAPs (Fermentable Oligo-, Di-, MonoSaccharides and Polyols) on Small and Large Intestinal Contents in Healthy Subjects Shown by MRI. American Journal of Gastroenterology 2014; 109: 110-119.

19. Marciani L, Foley S, Hoad CL, Campbell E, Armstrong A, Manby P, Gowland PA, Spiller RC. Effects of Ondansetron on small bowel water content: a magnetic resonance imaging study. Gut 2007; 56: A333.

400 20. Marciani L, Foley S, Hoad CL, Campbell E, Totman JJ, Cox E, Gowland PA, Spiller

401 RC. Accelerated small bowel transit and contracted transverse colon in diarrhoeapredominant irritable bowel syndrome (IBS-D): Novel insights from magnetic resonance imaging (MRI). Gastroenterology 2007; 132: A141-A141.

21. Placidi E, Hoad CL, Marciani L, Gowland PA, Spiller RC. Effects of an osmotic laxative on the distribution of water between the small and large intestine in humans. Gut 2010; 59(S1): A141. Chaddock G, Costigan C, Lam C, Hoad CL, de Vos WM, Gowland PA, Spiller RC. Mode of action of a macrogol formulation on distribution of Intestinal fluid: a MRI study. Gastroenterology 2012; 142: S814.

411 23. Lam C, Sanders D, Lanyon P, Garsed K, Foley S, Pritchard S, Marciani L, Hoad C, 412 Costigan C, Gowland P, Spiller R. Contrasting changes in small bowel water content in 413 patients with diarrhoea: coeliac disease and scleroderma versus IBS and healthy controls. United European Gastroenterology Journal 2013; 1: A108.

24. Froehlich JM, Patak MA, von Weymarn C, Juli CF, Zollikofer CL, Wentz KU. Small bowel motility assessment with magnetic resonance imaging. Journal of Magnetic Resonance Imaging 2005; 21: 370-375.

25. Fidler JL, Guimaraes L, Einstein DM. MR Imaging of the Small Bowel. Radiographics 2009; 29: 1811-1826.

420 26. Siddiki H, Fidler J. MR imaging of the small bowel in Crohn's disease. European Journal of Radiology 2009; 69: 409-417.

27. Gutzeit A, Patak MA, von Weymarn C, Graf N, Doert A, Willemse E, Binkert CA, Froehlich JM. Feasibility of small bowel flow rate measurement with MRI. Journal of Magnetic Resonance Imaging 2010; 32: 345-351.

28. Hahn T, Kozerke S, Schwizer W, Fried M, Boesiger P, Steingoetter A. Visualization and Quantification of Intestinal Transit and Motor Function by Real-time Tracking of F-19 Labeled Capsules in Humans. Magnetic Resonance in Medicine 2011; 66: 812-820.

29. Ajaj W, Goehde SC, Papanikolaou N, Holtmann G, Ruehm SG, Debatin JF, Lauenstein TC. Real time high resolution magnetic resonance imaging for the assessment of gastric motility disorders. Gut 2004; 53: 1256-1261.

431 30. Lauenstein TC, Ajaj W, Narin B, Gohde SC, Kroger K, Debatin JF, Ruhm SG. MR Imaging of apparent small-bowel perfusion for diagnosing mesenteric ischemia: feasibility study. Radiology 2005; 234: 569-575.

31. Marciani L. Assessment of gastrointestinal motor functions by MRI: a comprehensive review. Neurogastroenterology and Motility 2011; 23: 399-407.

32. Pritchard SE, Marciani L, Garsed KC, Hoad CL, Thongborisute W, Roberts E, Gowland PA, Spiller RC. Fasting and postprandial volumes of the undisturbed colon: normal 
438 values and changes in diarrhea-predominant irritable bowel syndrome measured using serial 439 MRI. Neurogastroenterology and Motility 2014; 26: 124-130.

440 33. Hahnemann ML, Nensa F, Kinner S, Gerken G, Lauenstein TC. Motility Mapping as 441 Evaluation Tool for Bowel Motility: Initial Results on the Development of an Automated 442 Color-Coding Algorithm in Cine MRI. Journal of Magnetic Resonance Imaging 2015; 41: 443 354-360.

444 34. Marciani L, Garsed KC, Hoad CL, Fields A, Fordham I, Pritchard SE, Placidi E, 445 Murray K, Chaddock G, Costigan C, Lam C, Jalanka-Tuovinen J, De Vos WM, Gowland PA, 446 Spiller RC. Stimulation of colonic motility by oral PEG electrolyte bowel preparation 447 assessed by MRI: comparison of split vs single dose. Neurogastroenterology and Motility 448 2014; 26: 1426-1436.

449 35. Bharucha AE, Fletcher JG, Seide B, Riederer SJ, Zinsmeister AR. Phenotypic 450 variation in functional disorders of defecation. Gastroenterology 2005; 128: 1199-1210.

451 36. Arkwright JW, Dickson A, Maunder SA, Blenman NG, Lim J, O'Grady G, Archer R, 452 Costa M, Spencer NJ, Brookes S, Pullan A, Dinning PG. The effect of luminal content and 453 rate of occlusion on the interpretation of colonic manometry. Neurogastroenterology and 454 motility : the official journal of the European Gastrointestinal Motility Society 2013; 25: e5245559. 


\begin{tabular}{|c|c|c|c|}
\hline Parameter & $\begin{array}{l}\text { mDIXON } \\
\text { (transit and } \\
\text { volumes) } \$\end{array}$ & $\begin{array}{l}\text { RARE } \\
\text { (water } \\
\text { content) }\end{array}$ & $\begin{array}{l}\text { High } \\
\text { Resolution AC } \\
\text { (positioning) }\end{array}$ \\
\hline $\mathrm{TR} / \mathrm{ms}$ & 3.0 & $\mathrm{~N} / \mathrm{A}$ & 2.4 \\
\hline $\mathrm{TE} / \mathrm{ms}$ & $\begin{array}{l}\mathrm{TE}_{1}=1.07 \\
\mathrm{TE}_{2}=1.9\end{array}$ & 400 & 1.2 \\
\hline $\mathrm{FA} /{ }^{\circ}$ & 10 & $\begin{array}{l}90 \\
\text { refocus } 108\end{array}$ & 42 \\
\hline $\begin{array}{l}\text { FOV / } \mathrm{mm}^{2} \\
\text { (Freq x Phase) }\end{array}$ & $250 \times 371$ & $400 \times 400$ & $330 \times 228$ \\
\hline Acq Resolution/ $\mathrm{mm}^{2}$ & $1.8 \times 1.8$ & $1.4 \times 1.76$ & $1.5 \times 1.5$ \\
\hline Recon Resolution/ $\mathrm{mm}^{2}$ & $0.98 \times 0.98$ & $0.78 \times 0.78$ & $0.86 \times 0.86$ \\
\hline $\begin{array}{l}\text { Slice thickness (gap) / } \\
\text { mm }\end{array}$ & 1.8 & $7(0)$ & $7(0.58)$ \\
\hline SENSE & 2.0 & 2.0 & 1.5 \\
\hline No. Slices & 111 & 20 & 8 \\
\hline No. Averages & 1 & 1 & 4 \\
\hline Orientation & Coronal & Coronal & $\begin{array}{l}\text { Oblique } \\
\text { Sagittal }\end{array}$ \\
\hline
\end{tabular}




\section{FIGURE LEGENDS}

467 Figure 1. Tagging applied to the ascending colon. 'Tags' are the dark stripes across the 468 image. There is a small shift in position of these tag lines in regions of predominately fat 469 tissue compared to water tissue (grey arrow) (a) Typical sagittal image showing no 470 movement within the colonic chyme - tags are straight and intact (white arrow). (b) Typical sagittal image showing movement within the colonic chyme. Both tag distortion (white arrow) and smearing and reduction of tag intensity (red arrow) due to movement are highlighted.

Figure 2. Processed images from tagged cine data showing (a) little motion (b) visible motion of colonic contents: (i) Mean pixel intensity map (calculated over 29 dynamic images). (ii) Corresponding pixel standard deviation map (calculated over 29 dynamic images). The displayed intensity scale for the standard deviation map is 5 times smaller than the mean pixel intensity map. The ascending colon region for each \%COV calculation is outlined in red.

Figure 3. (a) Individual data for MR free water in the small bowel (SBWC) at baseline, 60 and 120 minutes post ingestion. (b) Individual data for MR free water in the ascending colon at baseline, 60 and 120 minutes post ingestion. Statistically significant differences from baseline are shown (corrected for multiple comparisons).

Figure 4. Tagged images showing: (a) Simultaneous antegrade (red arrow) and retrograde flow (white arrow) in ascending colon. (b) The presence of a fast moving central retrograde 'jet' (grey arrow). (c) The reduction in tag intensity (red arrow) and complex flow in hepatic flexure of ascending colon (white arrow). 
493 Figure 5. Individual data for the calculated \% COV at baseline, 60 and 120 minutes post

494 ingestion. Statistically significant differences from baseline and between groups are shown 495 (corrected for multiple comparisons).

496 
Pritchard 23

497 FIGURES

$498 \quad 1 \mathrm{a}$

499

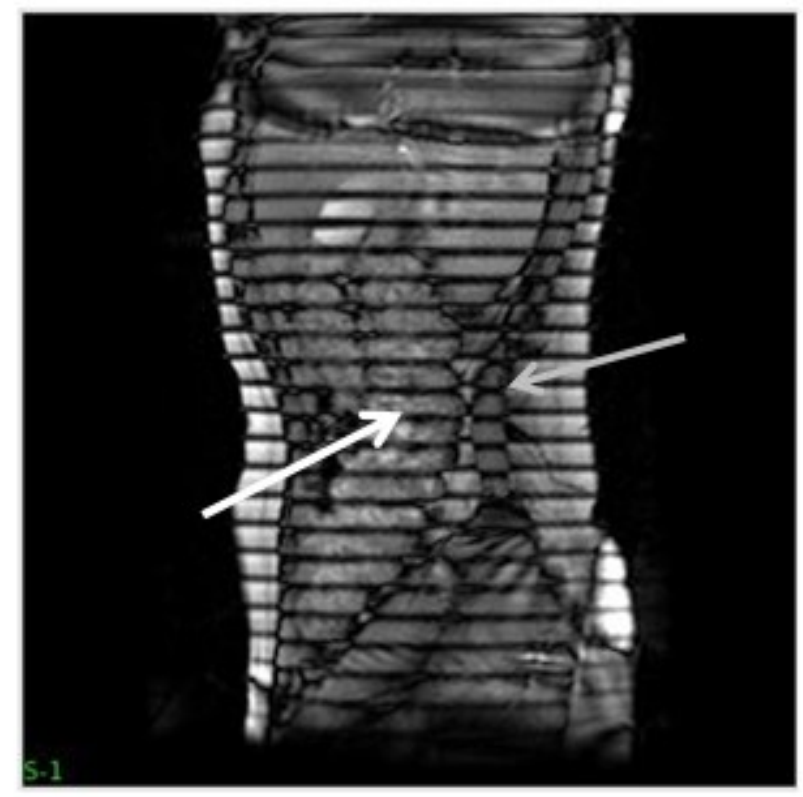

$500 \quad 1 b$

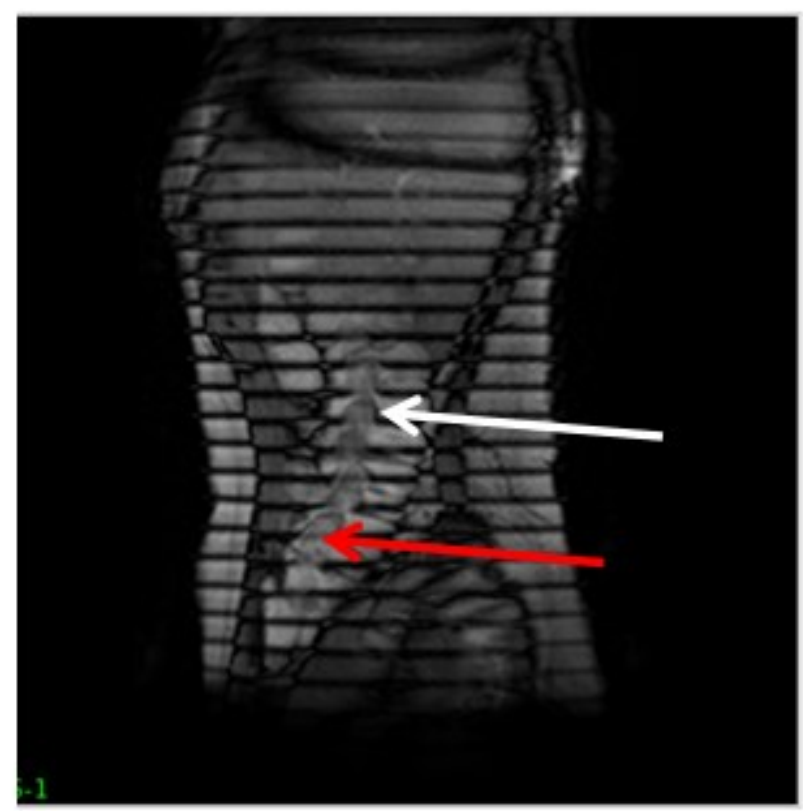

501

502 
Pritchard 24

$503 \quad 2 \mathrm{a}$

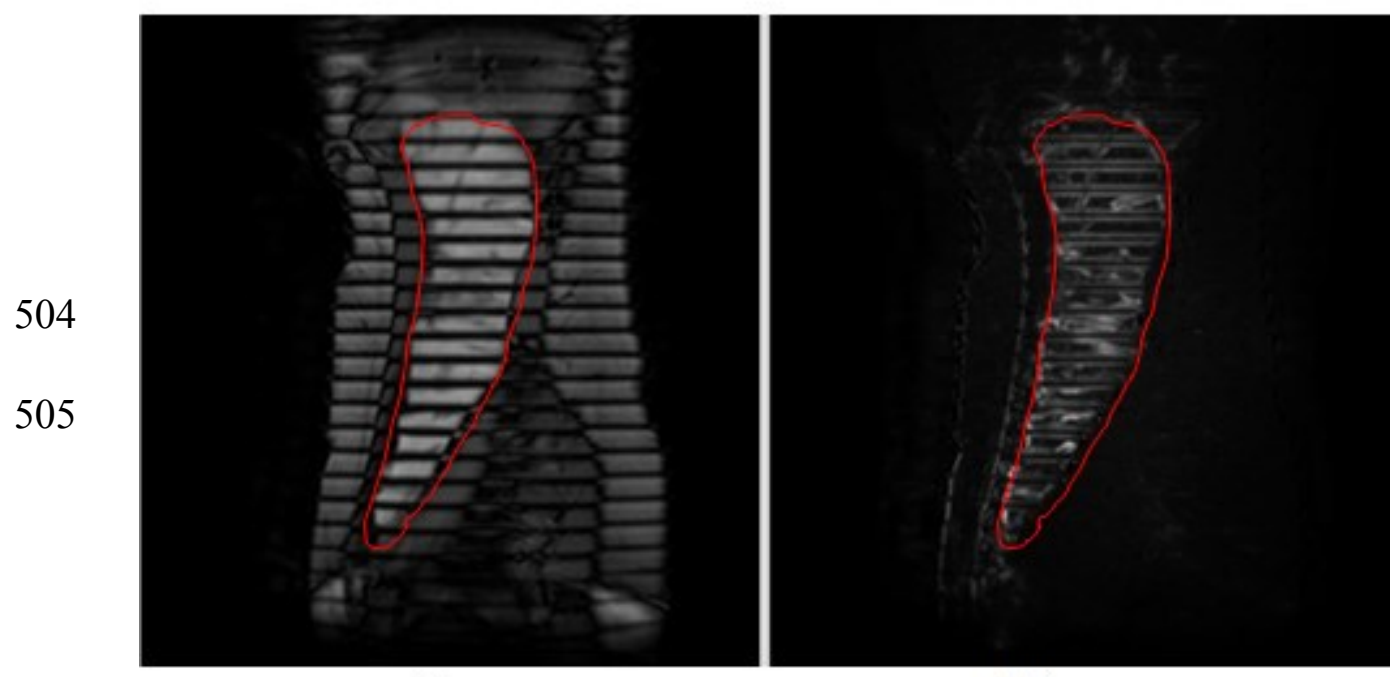

(i)

(ii)

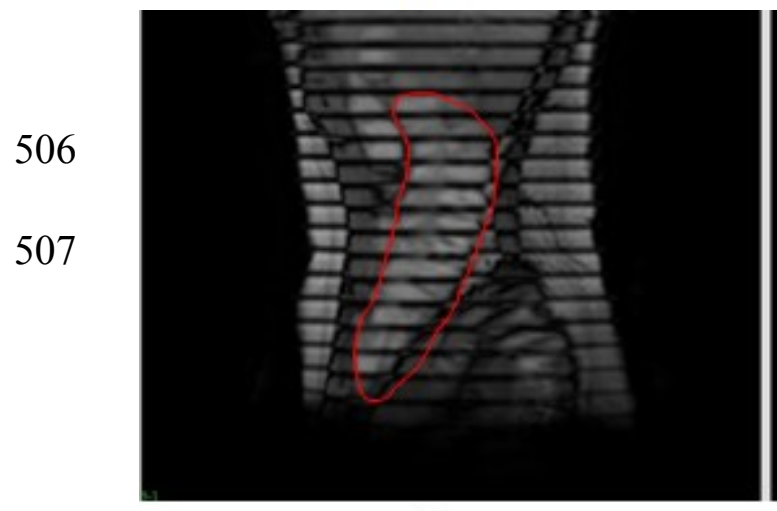

(i)

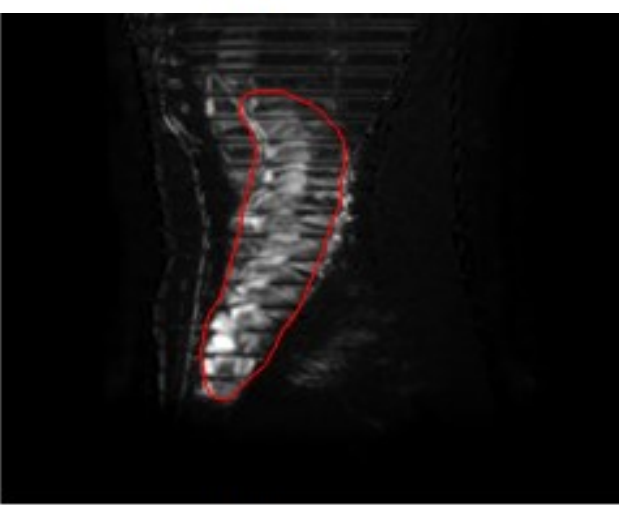

(ii) 
$508 \quad 3 a$

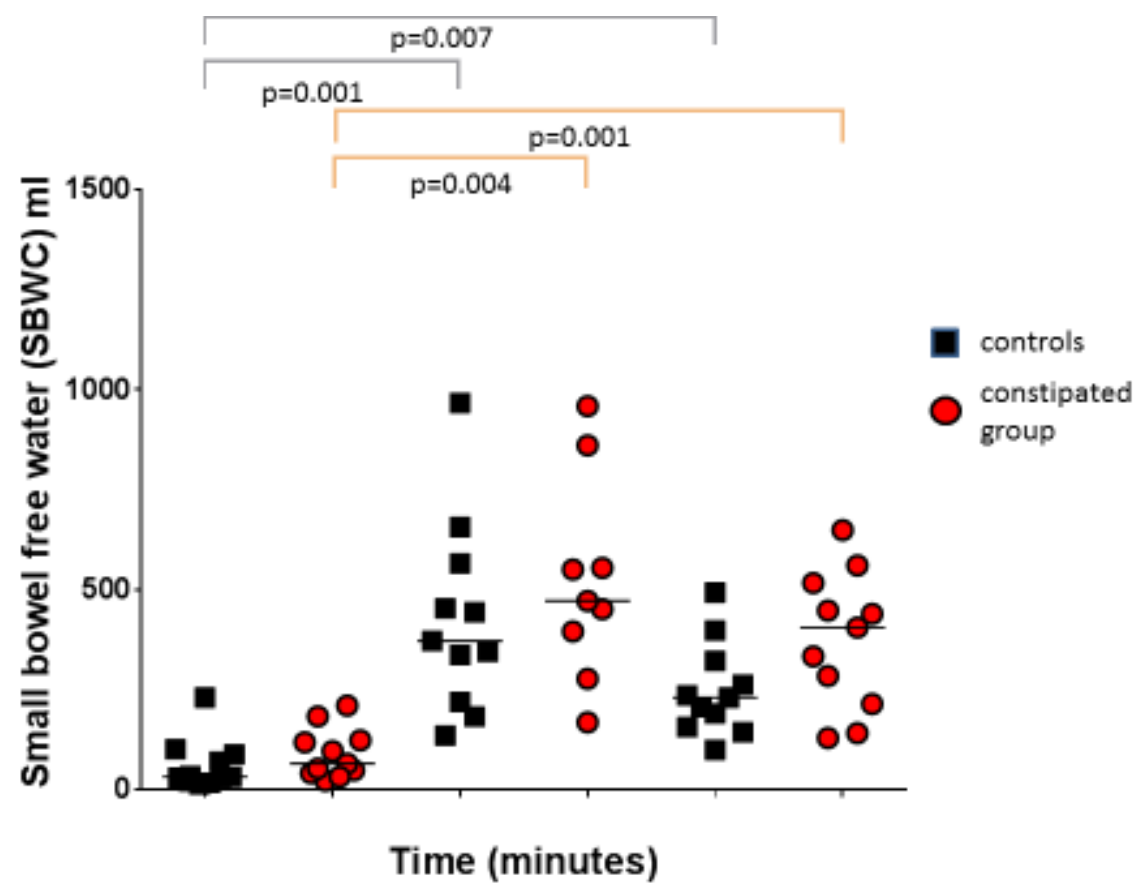

509

$5103 b$

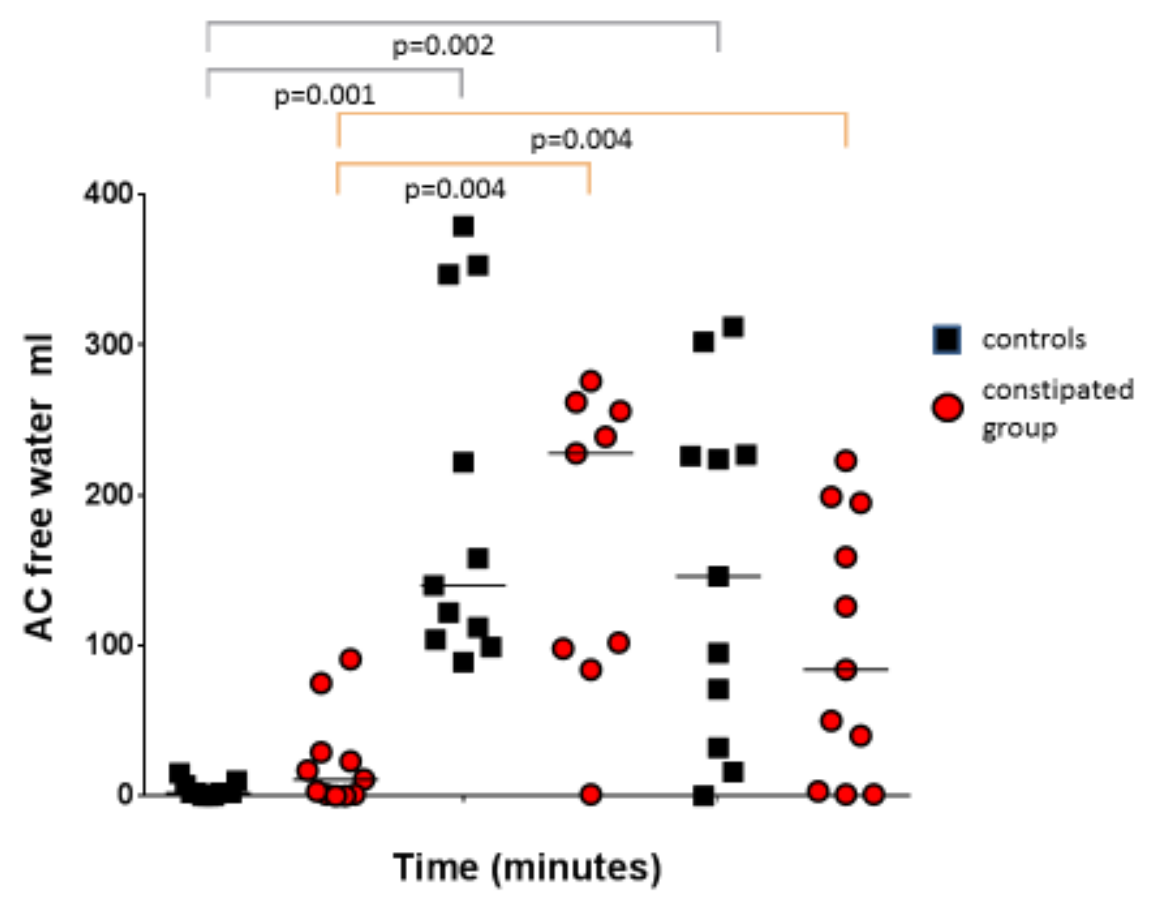


Pritchard 26

514

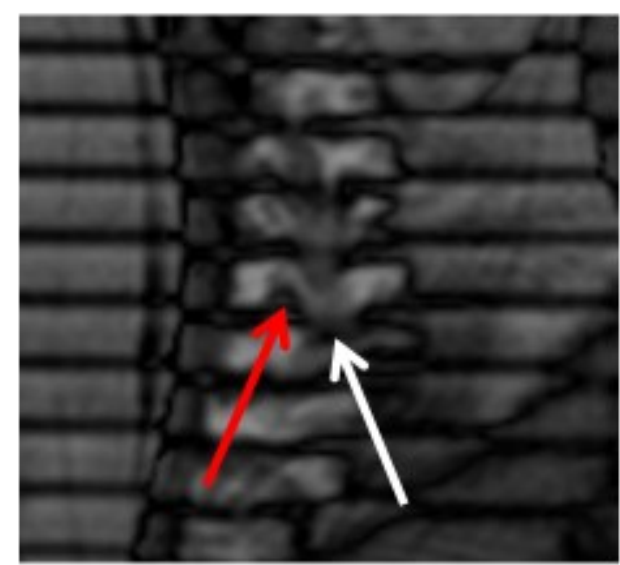

$515 \quad 4 b$

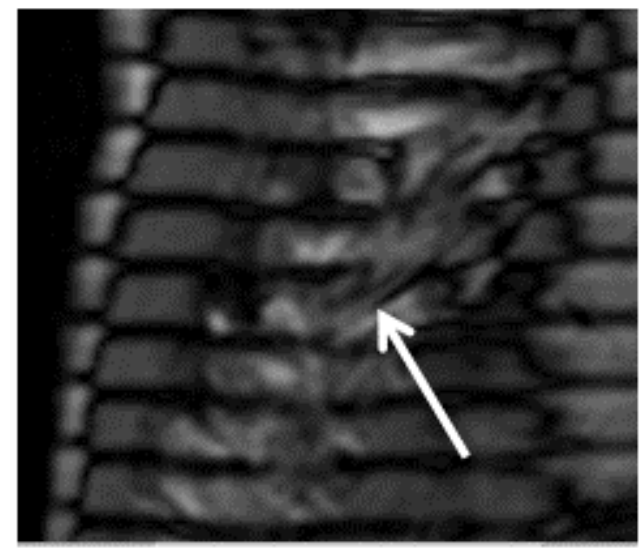

516

$517 \quad 4 c$

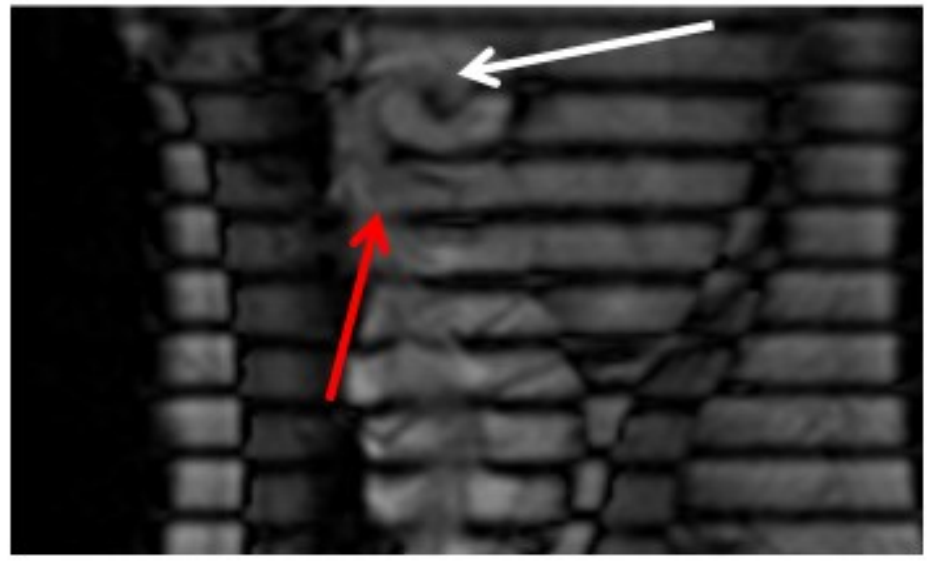

518

5195. 


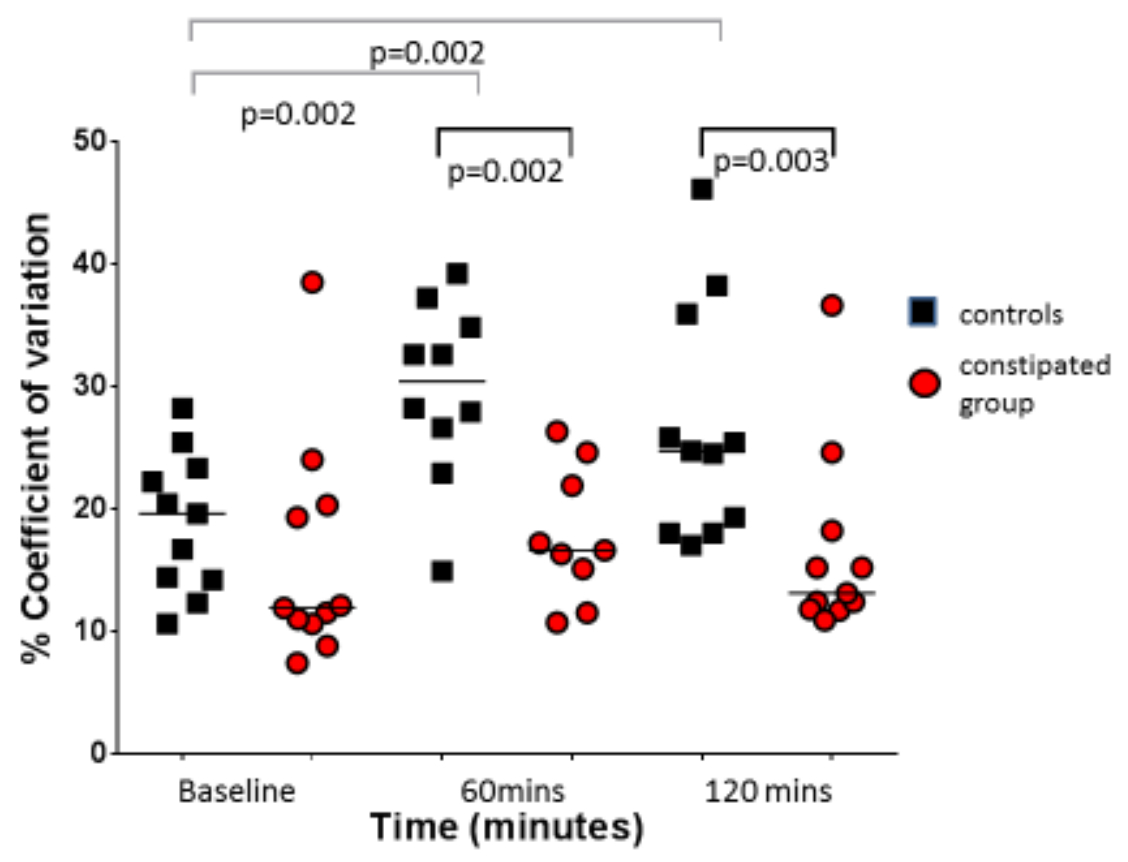

520

521 SUPPLEMENTARY INFORMATION

522 Video 1 An example of tagged images showing little motion in the fasted

523 ascending colon.

524 Video 2 An example of tagged images demonstrating motion in the fluid filled 525 ascending colon. 